\title{
Considering complexity: animal social networks and behavioural contagions
}

Josh A. Firth1,2,*

${ }_{1}^{1}$ Department of Zoology, University of Oxford, Oxford, UK, OX1 3PS

2Merton College, University of Oxford, Oxford, UK, OX1 4JD

*Correspondence: email joshua.firth@zoo.ox.ac.uk ; twitter @JoshAFirth

The spread of behaviours through animal social networks have often been considered as 'simple contagions'. However, research across other disciplines now provides substantial grounding for the 'complex contagion' of behaviours. The study of animal behaviour could benefit greatly from generally expanding to incorporate these new insights.

\section{Current Understanding of Behavioural Transmission in Animal Systems} The transmission of information in animal social systems takes place in many contexts such as through direct communication or inadvertent social cues, and it shapes various aspects of individuals' behaviour such as foraging or mating[1,2]. Social transmission is dependent upon the network of social connections between individuals, and these 'social networks' are increasingly acknowledged as fundamental for understanding social processes[1, 2, 3]. Furthermore, these socially-informed changes in behaviour have various implications, ranging from affecting individuals' fitness to shaping population-level processes[1, 2], and can even feedback to influence the structure of the social system and the subsequent spreading of behaviours[1].

To date, the majority of animal behaviour research examining this dynamic relationship between social networks and the transmission of behaviours has followed two related and widely-ascertained concepts. First, individuals that are more socially connected (i.e. more 'central') are more likely to gain and spread new behaviours [1, 2]. Second, adopting a new behaviour may dynamically feedback and increase an individual's social centrality, and this can then positively promote further transmission to others[1].

\section{The Complex Nature of the Social Diffusion of Behaviours}

The central assertions above, which are largely representative of the general stateof-knowledge across animal social network research[1], are underpinned with a simple assumption that is frequently (and sometimes unknowingly) made when considering the spread of behaviours. This is that new behaviours diffuse through social networks based on the 'total amount of social connections' i.e. the 'number of unique connections' for unweighted networks, or the 'number and the strength of the connections' for weighted networks[4]. Indeed, many studies often state that individuals with the most social connections are the most likely to gain, and spread, socially-informed behaviours $[1,2]$. Further, along with the qualitative assertion that increased social connectivity increases the potential for behavioural transmission, the primary quantitative method for assessing the spread of 
behaviours, is Network Based Diffusion Analysis (NBDA). This progressive

40 approach has provided much insight into social systems ranging from captive fish and insects, to wild birds and mammals[3, 4]. NBDA estimates the influence of a

48 The notion of the simple contagion of behaviours was initially formalised in likelihood of adopting the behaviour is related to the total amount of relevant network connections to 'informed' individuals [3, 4]. This assumption is known as a 'simple contagion', and is analogous to how a disease may spread through a network based simply on contacts between individuals[5, 6, 7]. sociology, where it was subsequently enormously influential[5, 6]. Yet, an increasing number of empirical studies, particularly from the growing field of computational sociology, are now consistently demonstrating that behaviours may generally spread as 'complex contagions' (Figure 1 and Box 1). The complex contagion concept contrasts with simple contagions. This alternative notion posits that socially-induced behavioural change does not simply depend on an individual's total amount of connections to informed individuals, but instead holds some 'complexity' $[5,6]$. This complexity, for instance, often comes from considering behavioural change in relation to some function associated with the proportion of individuals' social connections directed towards informed individuals. Therefore, the likelihood of adopting a behaviour often depends on the individual's exposure to both informed, and uninformed, individuals - instead of the simply the sum of connections with informed individuals (Figure 1).

Recent support for the complex contagion of behaviour comes from contexts as varied as human health, technological innovations, political movements, and even social media. Importantly, these empirical examinations of the spread of behaviours have often directly contradicted the patterns anticipated from simple contagions (i.e. simply that increased social connections will increase an individuals' propensity to gain and transmit new behaviours) $[5,6,7,8]$, and shown that 'disease spread' is a flawed metaphor for behavioural transmission[5]. A particularly compelling example comes from disease science itself; the spread of viruses (such as influenza, or HIV) can be explained relatively well by simple models, but the spread of anti-infection behaviours (such as vaccinations, or HIVprevention measures respectively) are not. Instead, adoption of these behaviours are instead based on more complex social factors, and thus they are likely spread as 'complex contagions' $[5,6,7]$. This shift in the basic expectations of contagions (from simple to complex) holds important ramifications for considering the spread of behaviours, both in terms of individuals' adoption of a behaviour, and how they may potentially feedback to shape the social structure and subsequent spreading pattern $[5,6,7,9]$ (see Box 1). 


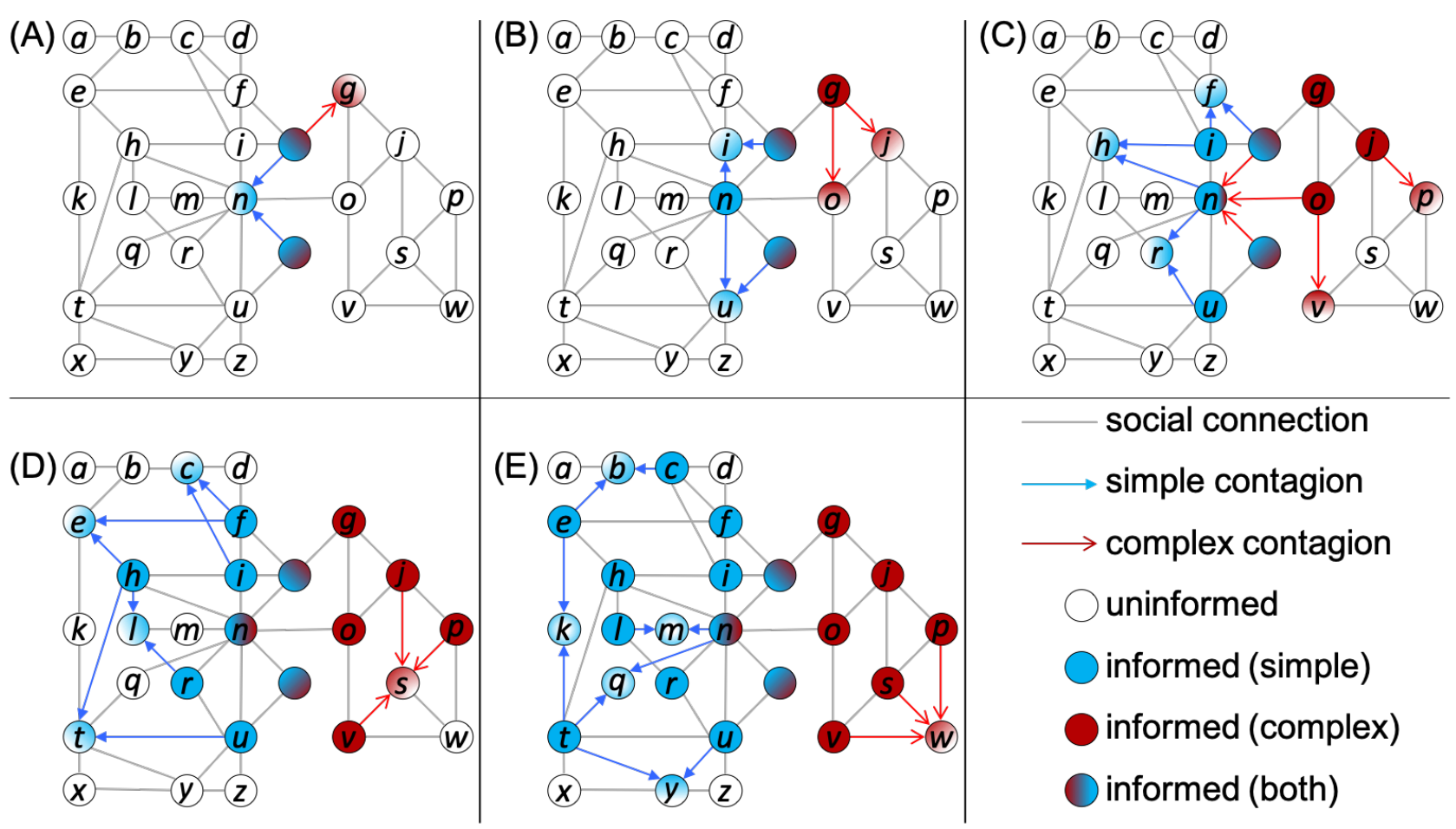

Figure 1. Simple and complex contagions on social networks.

Examples (A-E) using undirected unweighted social networks to show how simple contagions can differ from complex contagions. The likelihood of up-taking the simple contagion behaviour ( $\underline{S}$-blue) is based simply on the total amount of social ties to 'informed' individuals (most ties = most likely). The likelihood of up-taking this complex contagion behaviour example $(\underline{C}-$ red) is based on the proportion of ties to informed individuals (highest proportion $=$ most likely). Here, these independent contagions are based entirely on these rules alone, so $\underline{S}$ spreads primarily through the highly connected individuals (left in each panel) and $\underline{C}$ spreads through the clustered individuals (right), demonstrating interesting differences: (A) Beginning with two 'starting individuals' (the red/blue individuals holding $\underline{S}$ and $\underline{C}$ ), individual $n$ (the most 'central' with most connections) has the most connections to the starting individuals and therefore most likely to uptake $\underline{S}$, while individual $g$ is most likely to uptake $\underline{C}$ due to having the highest proportion (1/3) of connections to the starting individuals (despite less overall connections to them). (B) $\underline{S}$ is most likely to spread from the starting individuals, or individual $n$, to the other relatively central individuals $i$ and $u$, while the more peripheral individuals $j$ and $o$ are most likely to uptake $\underline{C}$ due to higher proportions of connections to $\underline{C}$-informed individuals. (C) Only now does $n$ (the most central individual) become one of the most likely to uptake $\underline{C}$ ( $n, p$, and $s$ all have a third of their connections to $\underline{C}$-informed individuals). (D) $n$ is not highly influential in spreading $\underline{C}$ (unlike $n$ was with $\underline{S}$ ), as $n$ 's associates are relatively central so only a relatively low proportion of their connections are $\underline{C}$-informed, and $\underline{C}$ instead is most likely to spread to the more clustered individual $s$. (E) Most relatively central individuals are $\underline{S}$ informed and only peripheral individuals remain unlikely to uptake $\underline{S}$, while $\underline{C}$ becomes more established within cliques (e.g. all of $w^{\prime}$ s connections are $\underline{C}$-informed). As such, in $\mathbf{A}-\mathbf{E}$, the two independent contagions differ in which individuals are likely to uptake the behaviours and subsequently influence their spreading. 
110 Box 1. Altering Expectations: Simple vs. Complex Contagions

111 The interplay between social connections and behavioural transmission[1], is altered when

First, the socially central individuals (that hold many dispersed social connections which bridge the network and are not constrained to cliques[2]) are considered particularly important for adopting behaviours and enabling rapid social spreading when the total amount of social connections is considered[1] i.e. for simple contagions. Yet, in complex contagions, these central individuals can instead be some of the least likely to initially adopt a new behaviour if - for instance - a high proportion of their social connections are directed to those not expressing the behaviour [5, 10] (Figure 1a-1c). Furthermore, even if these central individuals do adopt the behaviour early, they are less likely to facilitate its spread in a complex contagion if - for instance - behavioural adoption by others depends on multiple social exposures within cliques [5, 6, 8] (Figure 1d-e).

Second, a dynamic relationship between sociality and behavioural transmission may exist, whereby adopting a behaviour causes individuals to gain new social connections[1]. This feedback would be predicted to promote the spread of behaviour under the expectations of simple contagions. However, changes in social connections that aid simple contagions can actually hinder complex ones. New sociological research shows that increasing individuals' social connections to non-informed individuals from other social groups can instead decelerate complex contagions[5]. For instance, if newly-informed individuals retain their current connections (rather than gaining new connections to non-informed individuals) they have an increased likelihood of (i) continually expressing the behaviour and of (ii) their group establishing a high prevalence of informed individuals and promoting spread to all members[5]. In this way, maintaining clique-based social organisation can allow complex contagions to become reinforced within local neighbourhoods, and ultimately spread the behaviour more effectively [5, 8]. This is somewhat comparable to the spread of cooperation, where exposing cooperators to non-cooperators can cause cooperative behaviour to breakdown[9].

\section{Advancing Insights into how Animal Behaviours Spread}

Although potentially challenging, many of the separate tools and conceptual approaches required for developing a general understanding of the contagion of animal behaviours across natural populations already exist. Vast amounts of research on animal Social Learning Strategies (SLS)[3] have long presented compelling evidence that adopting socially-informed behaviours may depend on more than just social connections alone. Indeed, animals express various SLS governing who to learn from, and when[3,11]. For instance, experiments in wild birds have suggested that SLS, such as 'conformity' or 'learning from relevant tutors', may influence information diffusion through social networks[11, 12]. In this way, SLS can cause behaviours to spread as complex contagions as transmission is then not just governed by the social network, but also by the specific rule (i.e. the SLS) shaping the uptake of the behaviour from their social connections. Further, the specifics of the SLS will also determine how the spreading pattern differs from that expected from a simple contagion. For example, assessments in captive schooling fish have shown that even the spread of relatively basic behaviours (escape responses) may be markedly better explained by complex, rather than simple, contagions when uptake is based on the proportion, not sum, of individuals' neighbours expressing the behaviour[10]. 
161 It is now important to move forward from generally just considering sociallytransmittable behaviours to diffuse as simple contagions through wild animal populations. Instead, the unique opportunities that these natural systems present should be utilised to create an ideal framework for directly examining the factors causally shaping behavioural spread across social networks. Specifically, recent but largely independent - advances in animal social networks provide the opportunity: (i) to experimentally create specified social network structures[11], (ii) observe the fine-scale spread of seeded behaviours [10,12] and (iii) how this feeds back onto social connections[1], and (iv) to statistically quantify the influence of social structure and specific SLS[4,12] on the dynamics of contagions. Developing and combining these complementary approaches will allow simultaneous quantification of how different behavioural rules, SLS and network architectures contribute to the spread of behaviour (and how this feeds back onto social structure itself) in natural populations.

As such, integrating the complex contagion concepts into the rapidly developing field of animal social networks and social learning would markedly contribute to understanding of social processes and diverse kinds of socially-transmittable behaviours (such as movements, foraging, sexual, anti-predatory etc). This will not just reveal how individual network positions and overall society structure relates to behaviour spread, but will also aid in establishing which behaviours diffuse differently/similarly to one another, how contrasting contagions may interact with each other, and the wider consequences of these contagions. Furthermore, other systems examining social contagions (e.g. within sociology) often lack some of the capabilities available within natural animal societies[2], such as being able to carry out real-world experiments or track many individuals in great detail across their lifetime and over many generations. As such, the ability to take such a comprehensive approach to testing behavioural transmission within animal social systems now also holds the potential to increase conceptual knowledge of complex contagions more generally across disciplines.

Much progress has recently been made in understanding the dynamic relationship between individuals' social connections and the adoption of behaviours in animal systems[1]. But, to build upon our understanding of sociality and to potentially advance investigations into contagions generally, we now need to explicitly incorporate the recent advances made within other disciplines [5] into the newly emerging approaches within animal behaviour, and consistently consider the potential complexity of behavioural contagions in animal social networks.

\section{References}

1. Kulahci, I.G. and Quinn, J.L. (2019) Dynamic Relationships between Information Transmission and Social Connections. Trends Ecol. Evol. 34, 545-554.

2. Kurvers, R.H.J.M., et al. (2014) The evolutionary and ecological consequences of animal social networks: emerging issues. Trends Ecol. Evol. 29, 326-335.

3. Hoppitt, W. and Laland, K.N. (2013) Social Learning An Introduction to Mechanisms, Methods, and Models. Princeton University Press. 
4. Hoppitt, W. (2017) The conceptual foundations of network-based diffusion

5. Centola, D. (2018) How Behaviour Spreads: The Science of Complex

6. Guilbeault, D., et al. (2018) Complex contagions: A decade in review. In Complex Spreading Phenomena in Social Systems (Lehmann, S. and Ahn, Y.Y., eds), pp. 3-25, Springer Nature.

7. Campbell, E. and Salathe, M. (2013) Complex social contagion makes

networks more vulnerable to disease outbreaks. Sci. Rep. 3.

8. Montanaria, A. and Saberib, A. (2010) The spread of innovations in social

networks. Proc Natl Acad Sci USA 107, 20196-20201.

9. Rand, D.G., et al. (2011) Dynamic social networks promote cooperation in experiments with humans. Proc Natl Acad Sci USA 108, 19193-19198.

10. Rosenthal, S.B., et al. (2015) Revealing the hidden networks of interaction in mobile animal groups allows prediction of complex behavioral contagion. Proc Natl Acad Sci USA 112, 4690-4695.

11. Firth, J.A., et al. (2016) Pathways of information transmission among wild songbirds follow experimentally imposed changes in social foraging structure. Biol. Lett. 12, 20160144.

12. Somveille, M., et al. (2018) Movement and conformity interact to establish local behavioural traditions in animal populations. PLoS Comp. Biol. 14, e1006647.

Acknowledgements

I would like to thank Andrea Stephens, Neeltje Boogert, and three anonymous reviewers for extensive feedback on previous versions of the manuscript. JAF was supported by a research fellowship from Merton College and BBSRC (BB/S009752/1) and acknowledges funding from NERC (NE/S010335/1). 